\title{
Building Infinitely Many Solutions for Some Model of Sublinear Multipoint Boundary Value Problems
}

\author{
Guy Aymard Degla ${ }^{1,2}$ \\ ${ }^{1}$ The Abdus Salam International Centre for Theoretical Physics (ICTP), 34151 Trieste, Italy \\ ${ }^{2}$ Institut de Mathematiques et de Sciences Physiques (IMSP), 01 BP 613 Porto-Novo, Benin \\ Correspondence should be addressed to Guy Aymard Degla; gdegla@ictp.it
}

Received 17 July 2015; Accepted 15 September 2015

Academic Editor: Maoan Han

Copyright (c) 2015 Guy Aymard Degla. This is an open access article distributed under the Creative Commons Attribution License, which permits unrestricted use, distribution, and reproduction in any medium, provided the original work is properly cited.

\begin{abstract}
We show that the sublinearity hypothesis of some well-known existence results on multipoint Boundary Value Problems (in short BVPs) may allow the existence of infinitely many solutions by using Tietze extension theorem. This is a qualitative result which is of concern in Applied Analysis and can motivate more research on the conditions that ascertain the existence of multiple solutions to sublinear BVPs. The idea of the proof is of independent interest since it shows a constructive way to have ordinary differential equations with multiple solutions.
\end{abstract}

\section{Introduction}

BVPs occur in most of the branches of sciences, engineering, and technology, for example, boundary layer theory in fluid mechanics, heat power transmission theory, space technology, and also control and optimization theory. Concretely, BVPs manifest themselves through the modelling of the motion of a particle under the action of a force, the diffusion of heat generated by positive temperature-dependent sources, the distribution of shear deformation in a beam formed by a few lamina of different materials, the deflection of a beam, and the transverse displacement of an elastically imbedded rail to a distributed transverse load, and so forth [1]. In particular higher order linear differential equations subjected to multipoint boundary conditions, of which we are concerned, arise in the modelling of many phenomena of physical or technological nature such as the deflection of a curved beam, the three-layer beam, and the steam supply control slide [2].

Let us base our terminology on those of Degla [3], Elias [4], and Coppel [5]. Let $n, m$, and $k_{1}, \ldots, k_{m}$ be positive integers such that $2 \leq m \leq \sum_{i=1}^{m} k_{i}=n$, and let $a=a_{1}<\cdots<$ $a_{m}=b$ be $m$ real numbers. We will denote by $P$ the Levin polynomial defined by $P(t)=\prod_{i=1}^{m}\left(t-a_{i}\right)^{k_{i}}$ and we will deal with disconjugate $n$ th-order differential operators on $[a, b]$ of the form

$$
L x:=x^{(n)}+p_{1}(t) x^{(n-1)}+\cdots+p_{n}(t) x,
$$

where the coefficients $p_{1}, \ldots, p_{n}$ are given real-valued continuous functions on $[a, b]$; for instance, $L x=x^{(n)}$. The disconjugacy of the higher order differential linear operator $L$ means that every nontrivial solution of the ordinary differential equation $L x=0$ has less than $n$ zeros counting their multiplicities. This means also that $L$ has a Polya factorization; that is, there exist $n$ smooth positive functions $v_{i} \in \mathscr{C}^{n-i+1}([a, b]), 1 \leq i \leq n$, such that

$$
\begin{aligned}
& L x=v_{1} \cdots v_{n} D \frac{1}{v_{n}} D \cdots D \frac{1}{v_{1}} x \\
& \text { for every } x \in \mathscr{C}^{n}([a, b]),
\end{aligned}
$$

where $D=d / d t$ (cf. [5]).

It follows that $L$ admits Green's function associated with the Boundary Value Problems:

$$
\begin{aligned}
L x & =0, \\
x^{(j)}\left(a_{i}\right) & =0, \quad 1 \leq i \leq m, 0 \leq j \leq k_{i}-1,
\end{aligned}
$$


and so for every $f \in \mathscr{C}([a, b])$, there exists a unique solution of the BPVs:

$$
\begin{aligned}
L x & =f, \\
x^{(j)}\left(a_{i}\right) & =0, \quad 1 \leq i \leq m, 0 \leq j \leq k_{i}-1 .
\end{aligned}
$$

Besides, we will also adopt the notations $\|f\|_{\infty}=$ $\max _{a \leq t \leq b}|f(t)|$ and

$$
S_{f}(t)= \begin{cases}\frac{f(t)}{|f(t)|} & \text { if } f(t) \neq 0 \\ 0 & \text { if } f(t)=0\end{cases}
$$

for any $f \in \mathscr{C}([a, b])$.

Many authors have proved the existence of at least one nontrivial solution for sublinear Boundary Value Problems that can be transformed to the model problem

$$
L y=F(t, y)
$$

under various boundary conditions and where

$$
F:[a, b] \times \mathbb{R} \longrightarrow \mathbb{R}_{+}
$$

is sublinear with respect to $y$ uniformly on $t$; that is,

$$
\begin{aligned}
\lim _{|y| \rightarrow+\infty}\left(\max _{a \leq t \leq b} \frac{F(t, y)}{|y|}\right) & =0, \\
\lim _{y \rightarrow 0}\left(\min _{a \leq t \leq b} \frac{F(t, y)}{|y|}\right) & =+\infty ;
\end{aligned}
$$

see $[3,6-8]$ and the references therein. Some authors have shown the existence of multiple solutions (sometimes by introducing a parameter); see [9-15] and the references therein. But results about the cases of sublinear Boundary Value Problems of any order with infinitely many solutions are scarce; see $[15,16]$.

In this paper, using a new basic topological idea in the Theory of Differential Equations, we would like to underline that, under the hypothesis that $f$ is sublinear with respect to $y$ uniformly on $t$, infinitely many solutions may occur. This will be achieved by starting adequately with infinitely many functions satisfying the boundary condition and by constructing a sublinear (in fact bounded) function $F$ for which a sequence of these functions satisfies the Boundary Value Problem:

$$
\begin{aligned}
L y(t) & =F(t, y(t)), \quad a_{1} \leq t \leq a_{m}, \\
y^{(j)}\left(a_{i}\right) & =0, \quad i=1, \ldots, m ; j=0, \ldots, k_{i}-1 .
\end{aligned}
$$

\section{The Result}

We have the following.

Theorem 1. Let $\psi \in \mathscr{C}^{n}\left(\left[a_{1}, a_{m}\right]\right)$ be such that $L \psi>0$ everywhere in $\left[a_{1}, a_{m}\right]$ and satisfy the boundary condition of (4); for example, the unique solution $\psi$ of $L y=1$ and $y^{(j)}\left(a_{i}\right)=$ $0, i=1, \ldots, m ; j=0, \ldots, k_{i}-1$.
Moreover let $g \in \mathscr{C}^{n}\left(\left[a_{1}, a_{m}\right]\right)$ be such that

$$
\begin{aligned}
P(t) g(t)>0 & \text { for } t \in \bigcup_{i=1}^{m-1}\left(a_{i}, a_{i+1}\right), \\
g^{(j)}\left(a_{i}\right)=0 & \text { for } i=1, \ldots, m ; j=0, \ldots, k_{i}-1, \\
{[L g]\left(a_{i}\right)=0 } & \text { for } i=1, \ldots, m .
\end{aligned}
$$

(e.g., the function $g=\psi^{2 n+1}$ ).

Then there exist a positive integer $\ell_{0}$ and a bounded positive continuous function

$$
F:\left[a_{1}, a_{m}\right] \times \mathbb{R} \longrightarrow(0, \infty)
$$

such that all the functions $\psi+g / \ell$ with $\ell \geq \ell_{0}$ satisfy the single nonlinear problem

$$
L y=F(t, y)
$$

with the boundary condition of (4); that is,

$$
y^{(j)}\left(a_{i}\right)=0, \quad i=1, \ldots, m ; j=0, \ldots, k_{i}-1 .
$$

For a proof of this theorem, we will use the following.

Lemma 2 (see [3]). If $L$ is disconjugate, then there exists a continuous function $\varphi \in \mathscr{C}\left(\left[a_{1}, a_{m}\right]\right)$ positive on $\bigcup_{i=1}^{m-1}\left(a_{i}, a_{i+1}\right)$ with $\varphi /|P|$ having a positive infimum such that

$$
S_{P}(t) y(t) \geq \varphi(t)\|y\|_{\infty}, \quad a_{1} \leq t \leq a_{m},
$$

for every $y \in \mathscr{C}^{n}\left(\left[a_{1}, a_{m}\right]\right)$ satisfying the differential inequality

$$
L y \geq 0
$$

and the homogeneous Hermite m-point conditions

$$
y^{(j)}\left(a_{i}\right)=0, \quad 1 \leq i \leq m ; 0 \leq j \leq k_{i}-1 .
$$

Proof of Theorem 1. For the sake of simplicity we take $\psi$ to be the solution of

$$
\begin{aligned}
L x & =1, \\
x^{(j)}\left(a_{i}\right) & =0, \quad 1 \leq i \leq m, 0 \leq j \leq k_{i}-1,
\end{aligned}
$$

and so $\psi$ is $n$-times differentiable with

$$
\begin{aligned}
L \psi & =1>0, \\
\psi^{(j)}\left(a_{i}\right) & =0, \quad 1 \leq i \leq m, 0 \leq j \leq k_{i}-1,
\end{aligned}
$$

and furthermore, according to the above lemma, we have $\inf (\psi / P)>0$. Hence by setting $g=\psi^{2 n+1}$, it is clear that $P \cdot g$ is positive on $\bigcup_{i=1}^{m-1}\left(a_{i}, a_{i+1}\right)$ and moreover

$$
g^{(k)}\left(a_{i}\right)=0, \quad 1 \leq i \leq m, \text { and all } k=0, \ldots, n,
$$


which implies

$$
\begin{aligned}
& g^{(j)}\left(a_{i}\right)=0 \quad \text { for } i=1, \ldots, m ; j=0, \ldots, k_{i}-1, \\
& {[L g]\left(a_{i}\right)=g^{(n)}\left(a_{i}\right)+\sum_{j=0}^{n-1} p_{n-j}\left(a_{i}\right) g^{(j)}\left(a_{i}\right)=0} \\
& \text { for } i=1, \ldots, m .
\end{aligned}
$$

Now set

$$
\begin{aligned}
& \alpha:=\frac{1}{2} \min \left\{[L \psi](t), a_{1} \leq t \leq a_{m}\right\}=\frac{1}{2}, \\
& \beta_{0}:=\max \left\{[L \psi](t), a_{1} \leq t \leq a_{m}\right\}=1,
\end{aligned}
$$

and $\beta:=\alpha+\beta_{0}=3 / 2$.

Moreover, choose $\ell_{0} \geq 1$ such that $\left(1 / \ell_{0}\right)\|L g\|_{\infty} \leq \alpha=$ $1 / 2$. Thus for all $\ell \geq \ell_{0}$ we have, on the one hand,

$$
L y_{\ell}=L \psi+\frac{L g}{l} \geq L \psi-\frac{\|L g\|_{\infty}}{l} \geq 1-\frac{1}{2}=\frac{1}{2},
$$

and on the other hand,

$$
L y_{\ell}=L \psi+\frac{L g}{l} \leq \beta_{0}+\frac{\|L g\|_{\infty}}{l} \leq 1+\frac{1}{2}=\frac{3}{2},
$$

yielding

$$
\frac{1}{2} \leq L y_{\ell} \leq \frac{3}{2}
$$

Moreover let us set

$$
\begin{gathered}
\Gamma:=\{0\} \cup\left\{\frac{1}{\ell}, \ell \in \mathbb{N}, \ell \geq \ell_{0}\right\}, \\
y_{\mu}:=\psi+\mu g \text { for every } \mu \in \Gamma .
\end{gathered}
$$

Also set

$$
\begin{aligned}
A_{\mu} & :=\left\{\left(t, y_{\mu}(t)\right), a_{1} \leq t \leq a_{m}\right\} \quad \text { for each } \mu \in \Gamma, \\
A & :=\bigcup_{\mu \in \Gamma} A_{\mu} .
\end{aligned}
$$

Note at once that $A_{0}$ is the graph of $\psi$ and that, for every $\mu \in \Gamma$, $A_{\mu}$ is compact and so $A_{\mu} \backslash \bigcup_{i=1}^{m}\left\{\left(a_{i}, 0\right)\right\}$ is open in $A$.

For $\mu_{1} \neq \mu_{2}$ in $\Gamma$, we have

$$
\begin{aligned}
&(s, z) \in A_{\mu_{1}} \cap A_{\mu_{2}} \\
& \Longleftrightarrow z=y_{\mu_{1}}(s)=y_{\mu_{2}}(s) \\
& \Longleftrightarrow z=y_{\mu_{1}}(s),\left(\mu_{1}-\mu_{2}\right) g(s)=0, \\
& \text { with } \mu_{1} \neq \mu_{2}
\end{aligned}
$$

$$
\begin{aligned}
& \Longleftrightarrow z=y_{\mu_{1}}(s), g(s)=0, \\
& \Longleftrightarrow s \in\left\{a_{1}, a_{2}, \ldots, a_{m}\right\}, z=0 .
\end{aligned}
$$

Hence

$$
\begin{aligned}
A_{\mu_{1}} \cap A_{\mu_{2}}=\left\{\left(a_{i}, 0\right) ; i=\right. & 1, \ldots, m\} \\
& \text { for } \mu_{1}, \mu_{2} \in \Gamma \text { with } \mu_{1} \neq \mu_{2} .
\end{aligned}
$$

Besides it is not hard to see that $A$ is closed (in fact compact) because every sequence of elements of $A$ has a subsequence that is either contained in some fixed $A_{\mu_{0}}$ (which is compact as the graph of a continuous function on a compact set) or distributed into infinitely many $A_{\mu}$ in which case it has an adherent point in $A_{0} \subset A$.

Therefore by (29), (27), (24), and the continuity of the functions $y_{\mu}$, we have a well-defined and continuous map

$$
\begin{aligned}
& f: A \subset\left[a_{1}, a_{m}\right] \times \mathbb{R} \longrightarrow[\alpha, \beta] \subset(0, \infty), \\
& \text { where } \alpha=\frac{1}{2}, \beta=\frac{3}{2},
\end{aligned}
$$

characterized by the relation

$$
f(t, x):=L y_{\mu}(t) \quad \text { if }(t, x) \in A_{\mu} .
$$

But, by the well-known Tietze extension theorem (also known as Tietze-Uryshon-Brouwer theorem) [17, 18], this map $f$ has a continuous extension

$$
F:\left[a_{1}, a_{m}\right] \times \mathbb{R} \longrightarrow[\alpha, \beta] \subset(0, \infty) .
$$

It follows that for every $\mu \in \Gamma$ we have

$$
\begin{aligned}
L y_{\mu}(t) & =F\left(t, y_{\mu}(t)\right), \quad a_{1} \leq t \leq a_{m}, \\
y_{\mu}^{(j)}\left(a_{i}\right) & =0, \quad i=1, \ldots, m ; j=0, \ldots, k_{i}-1,
\end{aligned}
$$

completing the proof.

\section{Conflict of Interests}

The author declares that there is no conflict of interests regarding the publication of this paper.

\section{Acknowledgment}

The author would like to thank very much the Abdus Salam International Centre for Theoretical Physics (ICTP, Trieste, Italy) for its hospitality during his second visit as a Regular Associate.

\section{References}

[1] R. P. Agarwal, Boundary Value Problems from Higher Order Differential Equations, World Scientific, Singapore, 1986.

[2] M. Gregus, Third Order Linear Differential Equations, Mathematics and Its Applications, D. Reidel, 1987.

[3] G. A. Degla, "A unifying maximum principle for conjugate boundary value problems," Advanced Nonlinear Studies, vol. 1, no. 1, pp. 121-131, 2001.

[4] U. Elias, Oscillation Theory of Two-Term Differential Equations, vol. 396 of Mathematics and Its Applications, Kluwer Academic Publishers, Dordrecht, The Netherlands, 1997. 
[5] W. A. Coppel, Disconjugacy, vol. 220 of Lecture Notes in Mathematics, Springer, New York, NY, USA, 1971.

[6] L. H. Erbe and H. Wang, "On the existence of positive solutions of ordinary differential equations," Proceedings of the American Mathematical Society, vol. 120, no. 3, pp. 743-748, 1994.

[7] P. W. Eloe and J. Henderson, "Positive solutions for $(n-1$, 1) conjugate boundary value problems," Nonlinear Analysis, Theory, Methods \& Applications, vol. 28, no. 10, pp. 1669-1680, 1997.

[8] H. Brezis and L. Oswald, "Remarks on sublinear elliptic equations," Nonlinear Analysis. Theory, Methods \& Applications, vol. 10, no. 1, pp. 55-64, 1986.

[9] L. H. Erbe, S. C. Hu, and H. Wang, "Multiple positive solutions of some boundary value problems," Journal of Mathematical Analysis and Applications, vol. 184, no. 3, pp. 640-648, 1994.

[10] R. Ma, "Multiple positive solutions for nonlinear $m$-point boundary value problems," Applied Mathematics and Computation, vol. 148, no. 1, pp. 249-262, 2004.

[11] J. M. Davis, J. Henderson, and P. J. Y. Wong, "General lidstone problems: multiplicity and symmetry of solutions," Journal of Mathematical Analysis and Applications, vol. 251, no. 2, pp. 527548, 2000.

[12] R. I. Avery and J. Henderson, "Three symmetric positive solutions for a second-order boundary value problem," Applied Mathematics Letters, vol. 13, no. 3, pp. 1-7, 2000.

[13] Z. Liu and F. Li, "Multiple positive solutions of nonlinear two-point boundary value problems," Journal of Mathematical Analysis and Applications, vol. 203, no. 3, pp. 610-625, 1996.

[14] G. Degla, "Positive nonlinear eigenvalue problems for conjugate BVPs," Nonlinear Analysis: Theory, Methods \& Applications, vol. 55, no. 5, pp. 617-627, 2003.

[15] J. Coyle, P. W. Eloe, and J. Henderson, "Bifurcation from infinity and higher order ordinary differential equations," Journal of Mathematical Analysis and Applications, vol. 195, no. 1, pp. 3243, 1995.

[16] Q. Yao, "Existence, multiplicity and infinite solvability of positive solutions to a nonlinear fourth-order periodic boundary value problem," Nonlinear Analysis. Theory, Methods \& Applications, vol. 63, no. 2, pp. 237-246, 2005.

[17] J. Dugundji, Topology, Wm. C. Brown Company Publishers, Dubuque, Iowa, USA, 1989.

[18] H. Eschrig, Topology and Geometry for Physics, vol. 822 of Lecture Notes in Physics, Springer, Berlin, Germany, 2011. 


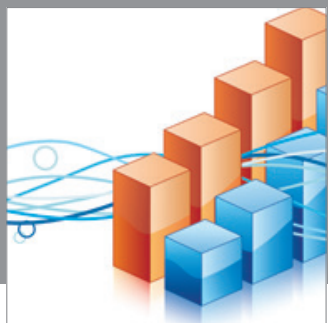

Advances in

Operations Research

mansans

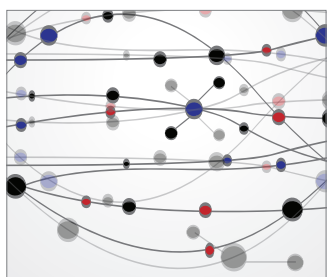

The Scientific World Journal
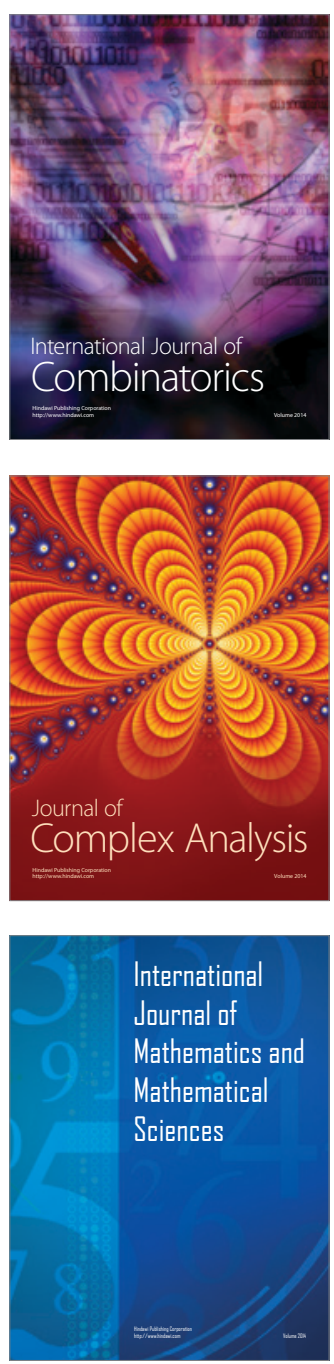
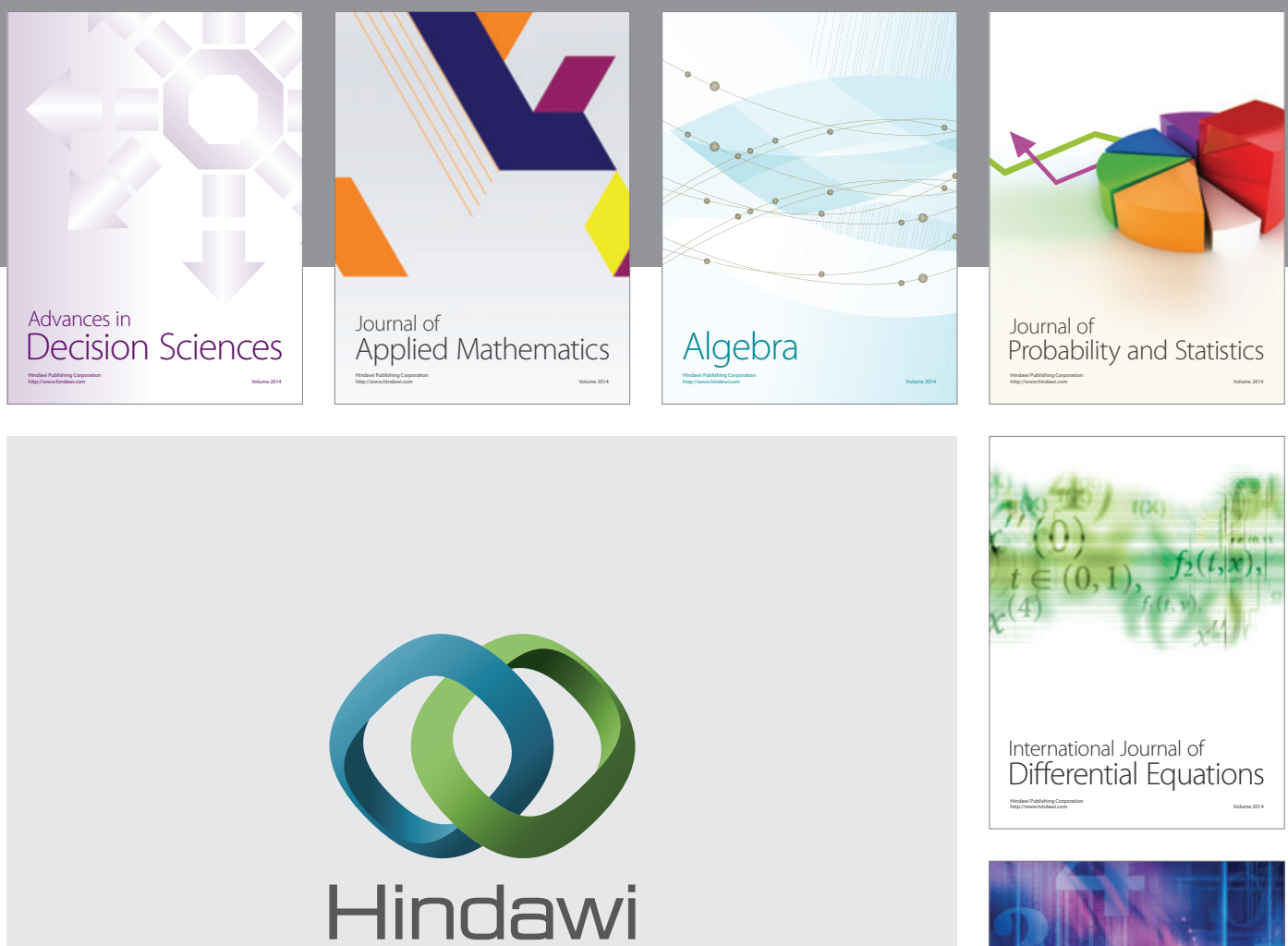

Submit your manuscripts at http://www.hindawi.com
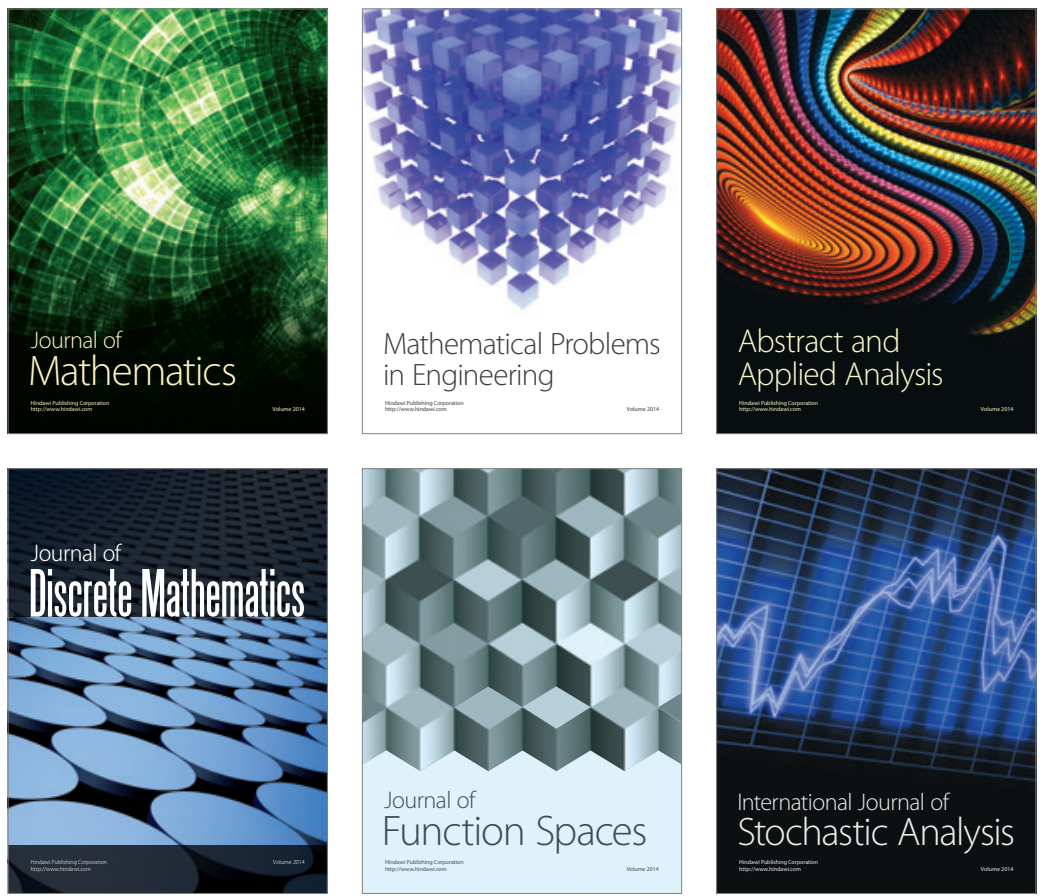

Journal of

Function Spaces

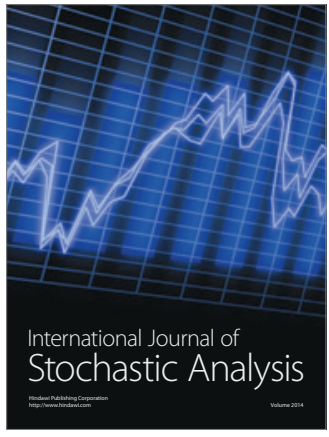

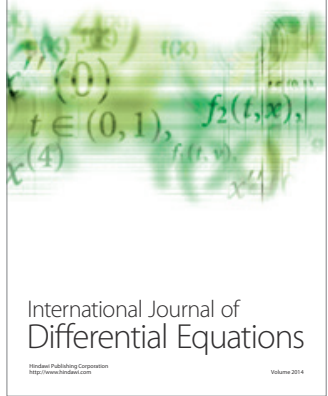
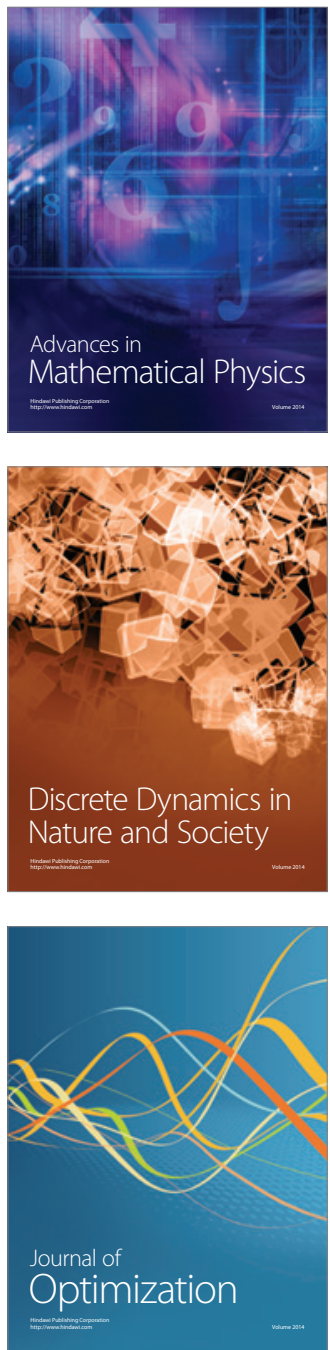